\title{
REGIONAL OBSTRUCTIVE EMPHYSEMA IN INFANCY
}

\author{
BY
}

\author{
JAMES THOMSON and JOHN O. FORFAR
}

From the Royal Infirmary, Dundee, and the Western General Hospital, Edinburgh, and from the Departments of Child Health, University of St. Andrews and University of Edinburgh

(RECEIVED FOR PUBLICATION JULY 31, 1957)

Over a quarter of a century ago Chevalier Jackson (1930) showed that the bronchi could act as valves in such a way that with respiration a lung or a lobe of a lung could become over-inflated. This valvular action would appear to be dependent on two physiological facts, first that the bronchi dilate on inspiration and contract on expiration (Reinberg, 1925; Heinbecker, 1927; Jackson, 1930), and that inspiration is predominantly an active muscular movement and is consequently more powerful than expiration which is largely a passive recoil. Thus on progressive reduction of the bronchial lumen by any obstruction a point will be reached at which the lumen is closed on expiration but allows air to enter on inspiration due to the greater power of this action and the slight bronchial dilatation which accompanies it. In the adult a functional disorder of this nature appears to be rare; in the infant, possibly due to the narrower bronchial lumen and greater elasticity of the bronchial wall, it can occur much more readily.

In generalized as opposed to regional obstructive emphysema the whole of the lung and both lungs are equally involved in the emphysematous process. Such may be seen in asthma or in acute bronchiolitis of infancy. In regional obstructive emphysema the emphysema is focal in distribution. It is more commonly unilateral but can be bilateral.

Valvular obstruction of the main lobar bronchus produces obstructive lobar emphysema in virtue of the fact that a lung lobe is a sealed self-contained unit (Fig. 1). Obstruction of smaller bronchi and bronchioles will not produce lobular emphysema provided the interalveolar pores (pores of Kohn) remain patent (Van Allen and Soo, 1933). In any segment of a lung less than a lobe these interalveolar pores allow escape of air from the alveoli in the affected segment to alveoli in the unaffected segment and thus prevent over-inflation in the affected segment. If, however, sticky secretions or other material or inflammatory swelling of the interalveolar septa cause obstruction of the interalveolar pores lobular emphysema may occur (Fig. 1). If, too, all the lobular bronchi are equally involved in the obstructive process so that no escape route for air remains, generalized lobular emphysema will result.

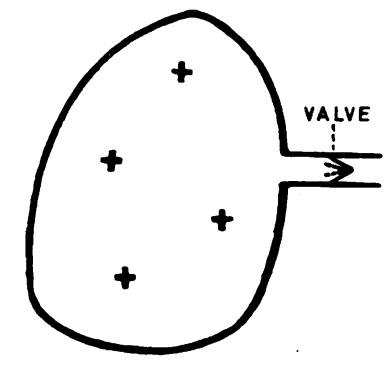

LOBAR OBSTRUCTIVE EMPHYSEMA
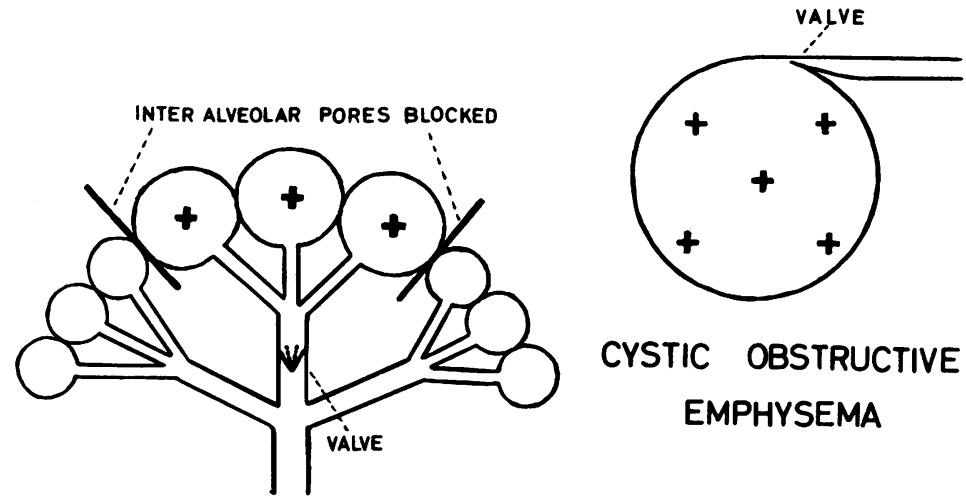

CYSTIC OBSTRUCTIVE EMPHYSEMA

\section{LOBULAR OBSTRUCTIVE}

\section{EMPHYSEMA}

FIG. 1.-Mechanism of production of obstructive emphysema-lobar, lobular and cystic. 
We report here a number of cases in which regional obstructive emphysema occurred in infants due to a variety of causes, and we classify the emphysema into three main anatomical types-lobar, lobular and cystic-with subsidiary groupings dependent on the pathogenesis of the condition.

\section{LOBAR OBSTRUCTIVE EMPHYSEMA}

\section{Congenital Lobar Emphysema: Presumed Bronchial Obstruction}

Case 1. This male infant, born at full term, was admitted to hospital on March 22, 1945, when 10 days old with the history that from the third day he had been having frequent breathless attacks. During these attacks he became cyanosed. Between attacks his breathing appeared normal. His birth had been normal.

On admission to hospital he was noted to be cyanosed and the respiration rate was rapid (60-70 per minute). The respirations were laboured. He had a cough, producing thin mucoid material. The percussion note over the left lung was hyper-resonant and over the right was impaired. On auscultation air entry was much diminished in all areas. The heart sounds were most distinctly heard on the right side of the chest.

Radiological examination revealed gross tension emphysema of the left lung, probably the left upper lobe with secondary collapse of the left lower lobe. The left side of the diaphragm was depressed, the left intercostal spaces widened and the mediastinum was displaced to the right (Fig. 2).

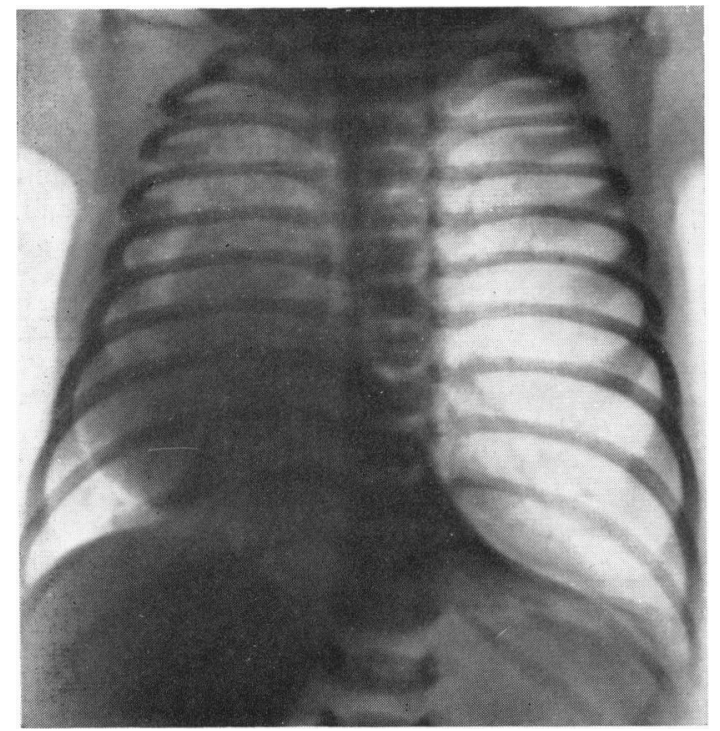

Fig. 2.-Case 1: Congenital lobar emphysema of the left upper lobe.

His condition deteriorated rapidly and he died a few hours after admission. No post-mortem examination was carried out.

\section{Congenital Lobar Emphysema: Presumed Bronchial Obstruction}

Case 2. A boy aged 4 weeks was admitted to hospital on September 26, 1955. He was the fourth child, born at full term after an uncomplicated labour lasting four hours. His birth weight was $9 \mathrm{lb} .5 \mathrm{oz}$. (4,198 g.).

Throughout his stay in a maternity unit his cry had been weak and his respirations noted to be somewhat laboured with accentuated respiratory movements. He was breast fed but required frequent rests during his feeds. He was discharged home on the tenth day.

On the fifteenth day of life he developed a sudden attack of cyanosis in which his lips were described as 'inky'. He held his head back and rolled it continuously from side to side. He did not lose consciousress and after one hour his colour gradually improved.

During the next two weeks he had several similar attacks of 10 to 60 minutes' duration. These bore no relation to feeding. For a week before admission he had a cough.

On admission he was a well-nourished baby with a weak cry. The temperature and pulse rate were normal. The respiration rate was 50 per minute. He had a slight cough and the alae nasi were moving. The chest remained fully expanded in all phases of respiration and the apex beat could not be detected. There was marked indrawing of the lower intercostal spaces and upper abdomen associated with vigorous respiratory efforts. Over the right lung the percussion note was hyperresonant and over the left it was relatively diminished. The breath sounds were much reduced on the right side and to a lesser extent on the left. Radiological examination showed well-marked obstructive emphysema of the right lung with displacement of the mediastinum to the the left (Fig. 3). The right lower and probably right

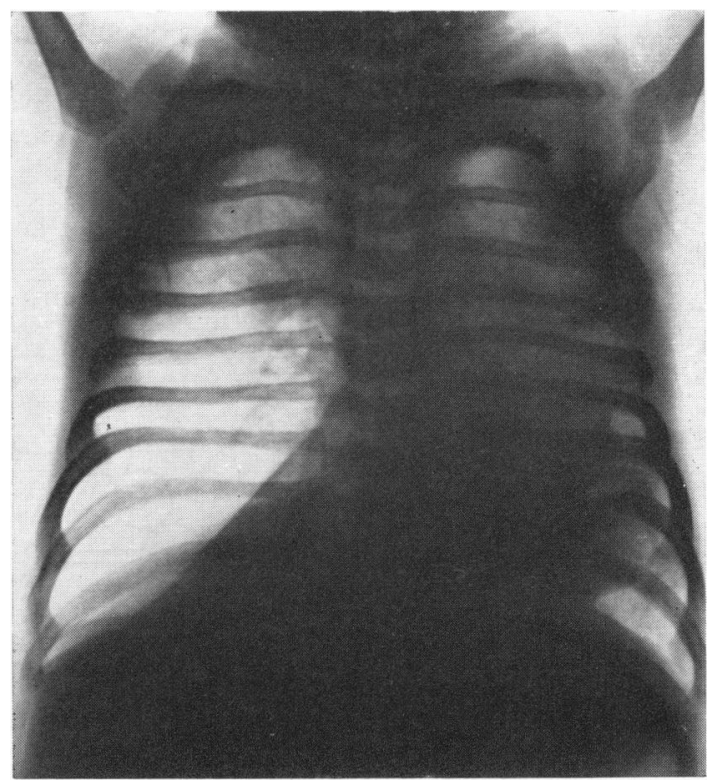

FIG. 3.-Case 2: Congenital lobar emphysema of the middle lobe of the right lung. 
upper lobes were subject to pressure collapse, suggesting that it was the middle lobe which was emphysematous. The intradermal tuberculin test was negative up to a strength of $1 / 1,000$.

On the second day after admission he had two brief cyanotic attacks accompanied by marked respiratory embarrassment and a severe degree of cyanosis not immediately relieved by oxygen.

Two days later bronchoscopy (Mr. M. Fallon) was carried out. During induction of anaesthesia and during the operation there was considerable respiratory difficulty. The left bronchus appeared normal. The right showed no evidence of a foreign body, but a bleb of pus was present in it and appeared to be coming from the right upper lobe bronchus.

On the following day he suddenly developed glottic oedema and required an emergency tracheotomy. Frequent suction was maintained through the tracheotomy tube and during this time there was no respiratory embarrassment. Thereafter the tracheotomy wound was allowed to close. When it did so episodic respiratory difficulty returned.

Radiologically right-sided emphysema persisted ; paradoxical movement of the mediastinum was not observed but absence of movement of the right side of the diaphragm was evident on screening.

Operative removal of the affected lobe was decided on, and was carried out at the age of 7 weeks. The operation was preceded by paracentesis through the chest wall, and the intrapulmonary pressure within the emphysematous lobe was found to be greater than the $35 \mathrm{~cm}$. of water which was the highest pressure which our manometer could record. The normal pressure at this age is said to be less than $5 \mathrm{~cm}$. of water. Deflation of the emphysematous lung facilitated anaesthesia. At operation the right middle lobe was seen to be grossly distended even in spite of decompression. It was resected. The adjacent lobes re-expanded immediately.

Post-operatively the child made a satisfactory recovery although he subsequently suffered an attack of pneumonia. Apart from that he has remained well over the past 18 months and radiologically the lung fields have remained normal.

Pathological examination of the excised middle lobe (Dr. K. Rhaney) showed that the whole of the right middle lobe was grossly over-inflated. Injected fluid passed freely in and out of the main bronchus. Cartilage in the walls of the bronchus appeared deficient but this may have been due to overdistension, giving a false appearance of disproportion. No mucous membrane folds or aberrant blood vessels likely to cause obstruction were found. Thus no convincing pathological cause for the obstruction could be demonstrated.

The possibility remains that deficiency of cartilage or some obstruction of the portion of the main bronchus not removed at operation was the responsible factor. It is possible also that functional distortion of the bronchus not evident in the excised lobe was present in life and contributed to the valvular obstruction.

\section{Congenital Lobar Emphysema : Presumed Bronchial Obstruction}

Case 3. This boy was born on September 3, 1956, some three weeks prematurely by spontaneous vertex delivery, birth weight $6 \mathrm{lb} .2 \mathrm{oz}$. $(2,778 \mathrm{~g}$.). He cried lustily after birth but the hands, feet, and face were cyanosed. A good deal of liquor was aspirated. Oxygen lessened but did not abolish the cyanosis. Some five hours after birth the respirations had become shallow and rapid (approximately 100 per minute). A few hours later there was marked costal margin recession and intercostal indrawing. It was noted that he was less distressed when lying on the right side. At that stage there was some diminution of air entry over the left lung. Radiologically the left lung was only slightly aerated, aeration of the right lower lobe was diminished and it was apparently normal in the right upper lobe. Despite this a lateral view showed anterior bowing of the sternum, suggesting emphysema, and well-marked aeration of the retrosternal space.

Three days later the respiration rate fell suddenly to 50-60 per minute, most of the symptoms disappeared and a chest film showed normal aeration of both lungs. Thereafter over a period of a month there were several episodes of sudden tachypnoea and slight cyanosis, not usually of longer than 15 minutes' duration. Periodic radiological examination showed varying degrees of collapse of the right middle and lower lobes. There was evidence of increased pressure within the right upper lobe with increased density of air, widening of the intercostal spaces and horizontal positioning of the ribs (Fig. 4). On screening the right upper lobe remained

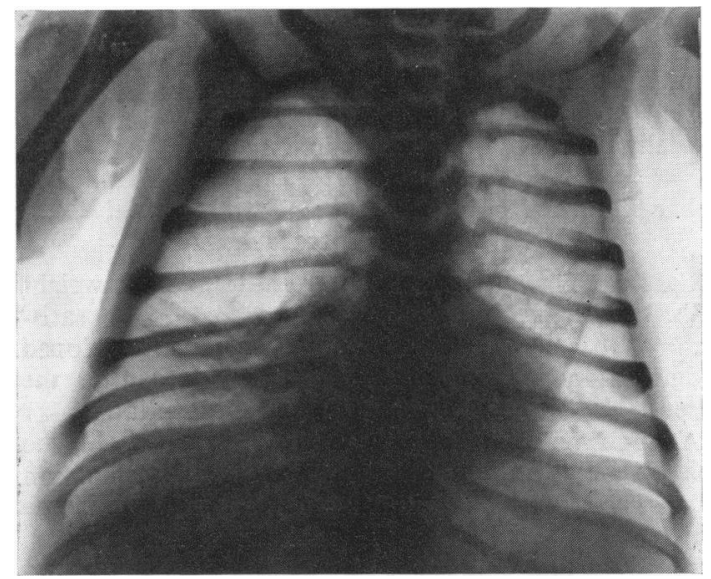

FIG. 4.-Case 3: Obstructive emphysema of the right upper lobe, showing horizontal position of ribs, widened intercostal spaces, and partial collapse of right middle and lower lobes.

inflated on expiration and herniated across the mediastinum (Fig. 5).

At the age of 2 months a bronchogram was carried out. Lipiodol freely entered the collapsed right lower and middle lobes showing no evidence of bronchial obstruction. The right upper lobe bronchus was filled much less satisfactorily despite attempts to accomplish. 


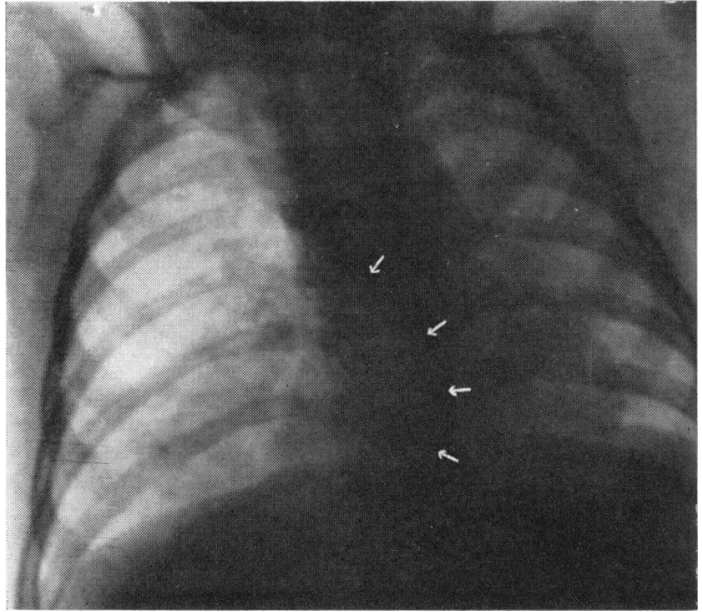

FIG. 5.-Case 3: Obstructive emphysema of the right upper lobe and mediastinal pleural herniation on expiration.

this. At this stage the air entry over the right upper lobe was markedly diminished.

This infant was considered to be suffering from congenital lobar emphysema of the right upper lobe. In the first few days of life this had prevented adequate expansion of the left lung and right lower lobe, presumably due to pressure on them, and latterly had caused pressure collapse of the right lower lobe.

After the age of 1 month his symptoms became less severe and he began to feed well and to gain weight satisfactorily. By the age of a year he was relatively symptom free, although he became severely distressed during an intercurrent respiratory infection. Radiologically there was little change in the lung.

\section{Lobar Obstructive Emphysema : Extrabronchial Com- pression by an Enlarged Heart}

Case 4. This girl had been born at term, birth weight $6 \mathrm{lb}$. $(2,722$ g. $)$. Her progress appeared to be satisfactory until she was 9 months old, when she developed cough, anorexia and fretfulness. Five days after the onset of these signs she was admitted to hospital.

At the time of admission, on November 8, 1948, she was relatively symptom free but radiological examination of the chest showed cardiac enlargement with some emphysema of the lower lobe of the right lung. There were no cardiac murmurs. Two days after admission there occurred a sudden incident of respiratory embarrassment when her respiration rate rose to 80 per minute, and the percussion note over the right lung became hyper-resonant as compared with the left. These signs disappeared after a few hours. For 10 days she remained well then developed, quite suddenly, a further episode of tachypnoea with respiratory distress. Clinically the heart was displaced to the left and there was intercostal indrawing. Radiological examination after the worst of her symptoms had passed off showed tension emphysema of the right middle and lower lobes (Fig. 6). A lateral film showed the trachea displaced backwards by the enlarged heart (Fig. 7).

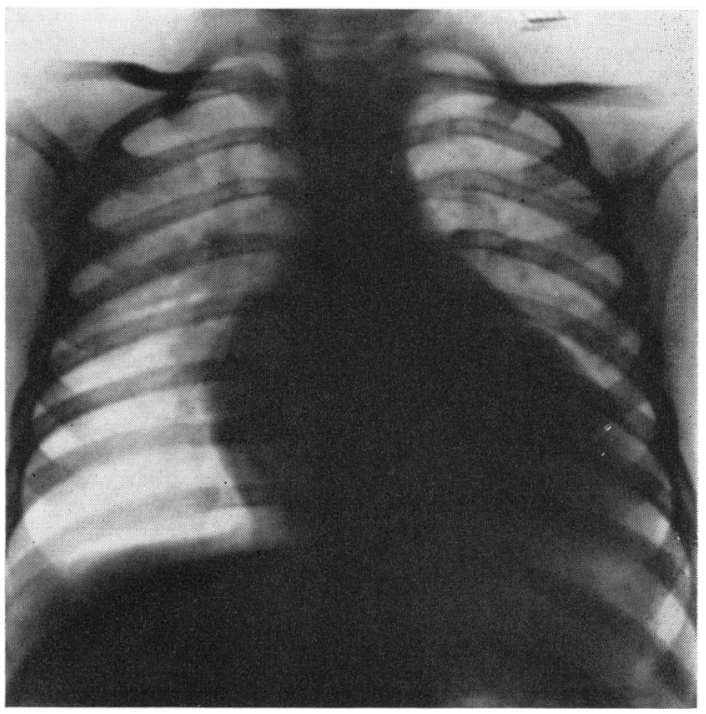

Fig. 6.-Case 4: Enlarged heart and obstructive emphysema of the right lower lobe.

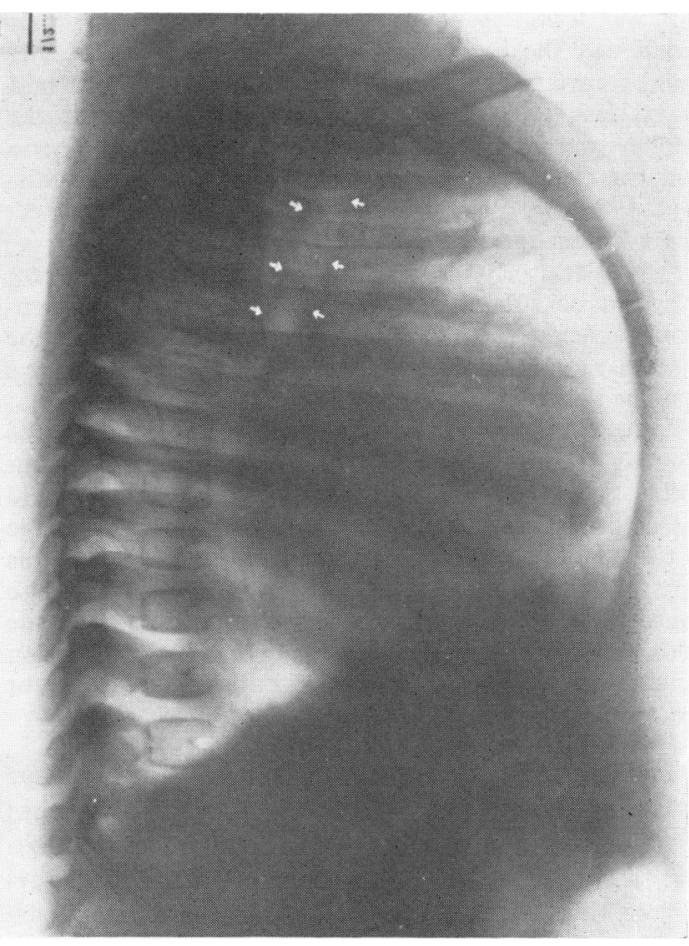

Fig. 7.-Case 4: Enlarged heart and backward displacement of the trachea (normally parallel with the vertebral column). 
Over the next few weeks a minor degree of emphysema of the right middle and lower lobes persisted. Finally the left lung collapsed suddenly and completely causing gross respiratory distress and she died 24 hours later.

At post-mortem examination the heart was enlarged $75 \%$ above the normal weight for this age. There were no valvular or septal defects. Unfortunately the heart was not examined histologically. The left lung was collapsed due to the pressure of the large heart on the left bronchus. The right lower lobe bronchus appeared liable to pressure from the same cause. There was emphysema of the right middle and lower lobes with partial collapse of the right upper lobe. Histological examination of the lungs confirmed the macroscopic findings.

Thus external pressure on the right lower lobe bronchus appeared to be the mechanism producing tension emphysema in this case.

\section{Lobar Obstructive Emphysema : Extrabronchial Com- pression by Enlarged Glands and Bronchial Obstruction by Mucopus}

Case 5. This boy, whose birth weight was $6 \frac{1}{2} \mathrm{lb}$. $(2,948 \mathrm{~g}$.), was a first child of a British mother and an Indian father. There was no family history of tuberculosis. At the age of $4 \frac{1}{2}$ months he was admitted urgently to hospital. For 11 days he had had a cough and wheezy respiration, and a few hours before admission to hospital he had become remarkably breathless and restless.

On admission on September 6, 1948, he showed wellmarked respiratory distress with intercostal indrawing and a respiration rate ranging from $\mathbf{4 0}$ to 60 per minute. The chest remained in the position of full inspiration. After a few hours his symptoms passed off but returned on the following day when there was diminished air entry over the lower lobe of the right lung with fullness of the right side of the chest. The percussion note over the right lung was resonant.

Radiological examination showed marked tension emphysema of the middle and lower lobes of the right lung, cardiac displacement to the left, depression of the right diaphragm, increase in the width of the intercostal spaces, upward displacement of the interlobar fissure and a mediastinal pleural hernia (Fig. 8). Radiological examination three days later when his acute symptoms had abated showed that the tension emphysema in the right lung had greatly diminished.

Over the ensuing weeks there was a progressive infiltration into the right lung with hilar gland enlargement. Bronchoscopy was carried out six weeks after admission. No foreign body was seen. A considerable amount of thick, sticky secretion was present in the right main bronchus and the mucosa was swollen, but no blockage could be detected. The left bronchus appeared normal. Two days after bronchoscopy a tension pneumothorax developed on the right side of the chest. This was relieved by removing $200 \mathrm{ml}$. of air which was under considerable pressure. $A$ week later he again developed a tension pneumothorax which, on this occasion, was accompanied by gross surgical emphysema extending from the neck to the lower limbs, and he died a few days later. The intradermal tuberculin test had been negative up to $1 / 100$ on two occasions a month apart.

At post-mortem examination there was mucopurulent secretion in the right lower lobe bronchus. A cavity was present at the right hilum but it was impossible to tell whether this was a liquefied lymph gland or a cavity in lung tissue. There was a septic bronchopneumonia with multiple abscess formation in the right lower lobe. There was also a cavity in the dorsal segment of the right lower lobe. The right lower lobe was covered with a fibrinopurulent layer and the remainder of the lung was adherent to the chest wall. Histological examination revealed a widespread tuberculous infection of the right lung and infection was also demonstrated in the left lung.

The mechanism producing the tension emphysema in this case appears likely to have been a combination of extrabronchial compression by tuberculous glands and intrabronchial obstruction by mucopurulent secretions. The latter appears to be the factor which precipitated the emphysema and which accounted for its sudden onset and disappearance.

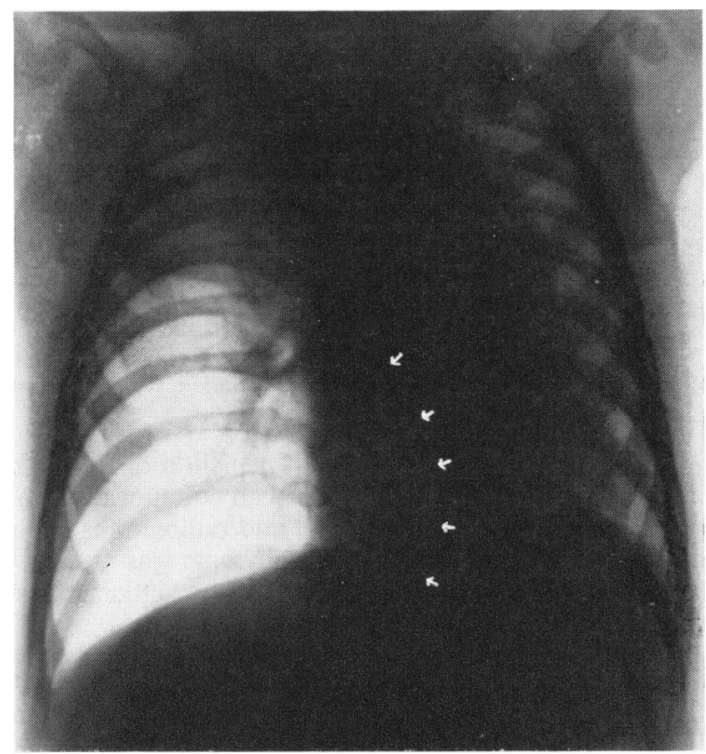

FIG. 8.-Case 5: Obstructive emphysema of the right lower lobe.

Lobar Obstructive Emphysema : Bronchial Obstruction by Mucopus

Case 6. This boy was born at home, birth weight $6 \mathrm{lb} .12 \mathrm{oz}$. (3,062 g.). He made satisfactory progress for the first four weeks. Five days before admission to hospital he developed an upper respiratory tract infection. Just before admission respiratory symptoms became much more marked with cough, cyanosis and increasing dyspnoea. 
On admission on February 13, 1957, his temperature was $101^{\circ} \mathrm{F}$. and his respiration rate 60 per minute. There was indrawing of the intercostal spaces and costal margin recession. The left side of the chest was fuller than the right. Coarse crepitations were audible over both lungs, especially the right. Cyanosis was evident and responded to oxygen. The white cell count was 14,000 per c.mm.

Radiological examination showed obstructive emphysema involving the left lung with some displacement of the mediastinum to the right and slight mediastinal pleural herniation, increased aeration of the left lung with widening of the intercostal spaces and depression of the diaphragm (Fig. 9). The right lung showed secondary collapse with patchy consolidation.

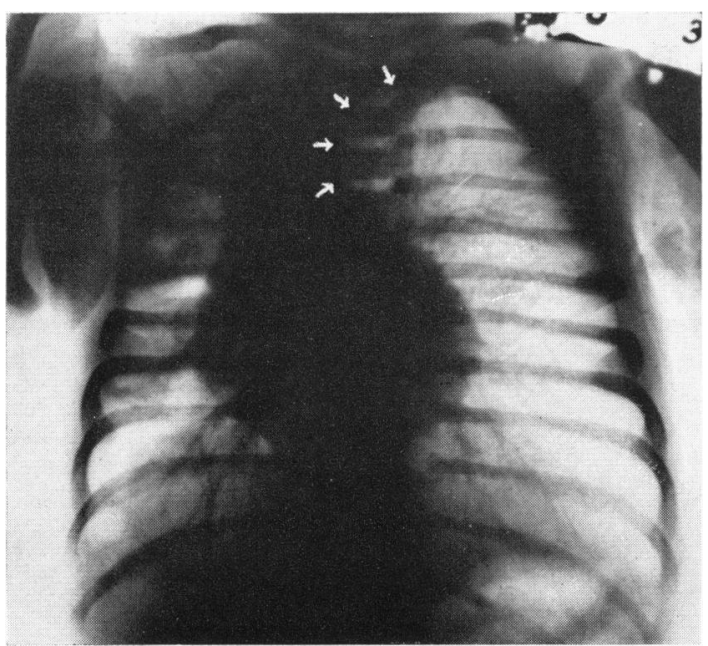

FIG.9.-Case 6: Obstructive emphysema of the left lung, mediastinal displacement, partial collapse and consolidation of the right lung.

After 48 hours' treatment with penicillin and streptomycin most of his symptoms had disappeared, his temperature had become normal and radiographs of the chest showed that obstructive emphysema was no longer present, the lungs showing only minor inflammatory change.

He made an uninterrupted recovery. It appears likely that the obstructive emphysema of the left lung resulted from obstruction of the left main bronchus with mucopus in an infant suffering from pneumonia.

\section{LOBULAR OBSTRUCTIVE EMPHYSEMA}

\section{Lobular Obstructive Fmphysema : Aspiration of Mucus and Spontaneous Pneumothorax}

Case 7. This boy was born at term on October 14 1956. He was very 'mucousy' at birth and was asphyxiated. He was given intragastric oxygen. He remained cyanosed and respiratory movement was accentuated.
Crepitations were audible over both lungs. Radiological examination just after birth showed patchy atelectasis throughout both lungs with an area of obstructive

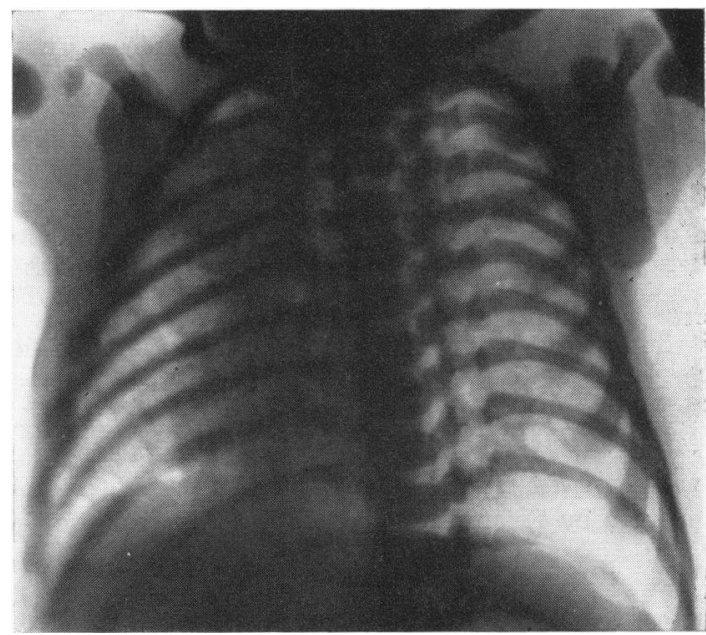

FIG. 10.-Case 7: Lobular obstructive emphysema of the left lower lobe.

emphysema at the left lung base (Fig. 10). Respiratory embarrassment continued and a second radiograph seven hours after the first showed a pneumothorax on the left side. Paracentesis of the left pleural cavity produced a few millilitres of air with some diminution of respiratory distress. On the following day marked respiratory embarrassment recurred and was relieved by aspirating the pleural cavity, when $20 \mathrm{ml}$. of air under considerably increased pressure were removed. Again there was marked clinical improvement. A few hours later the chest had again to be tapped. Over the next 24 hours his respiratory difficulty progressively diminished and the crepitations which had persisted from birth gradually disappeared. He made a satisfactory recovery and two days after birth there was no longer radiological evidence of pneumothorax.

Aspiration of mucus into the lungs in this case appeared to have produced obstructive emphysema involving particularly the lower lobe of the left lung. Air had ruptured into the pleural cavity from the over-distended left lung causing a pneumothorax.

Lobular Obstructive Emphysema : Aspiration of Mucus

Case 8. This boy was born at term on April 14, 1956, by spontaneous vertex delivery, the birth weight being $6 \mathrm{lb}$. 3 oz. $(2,806$ g.). He was very 'mucousy' at birth. Twelve hours after birth moderate respiratory difficulty was still present, the costal margin receding on inspiration and the anterior chest wall bulging forward. Radiological examination showed hyperaeration of the lungs, particu- 
larly on the left side with splaying and horizontal positioning of the ribs, depression of the diaphragm and some mediastinal displacement to the right (Fig. 11).

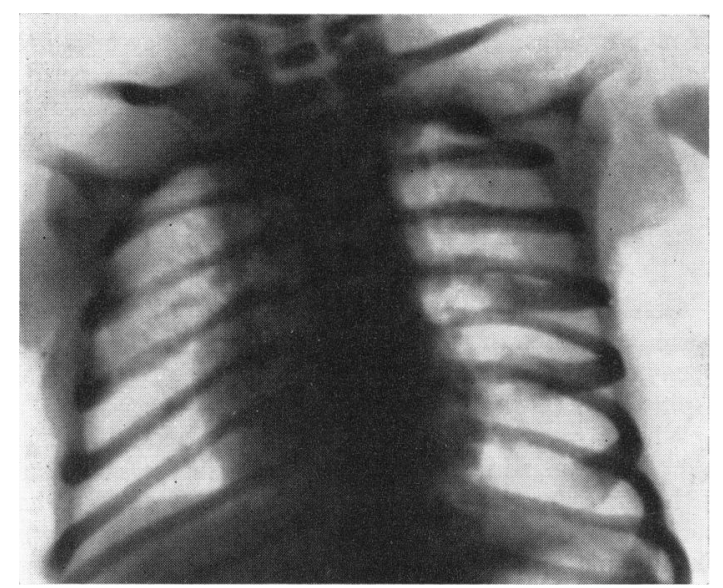

Fig. 11.-Case 8: Obstructive emphysema, especially of the left lung, widening of the rib spaces and slight mediastinal displacement.

A few days later all abnormal physical and radiological signs in the chest had disappeared.

In this case, consequent on aspiration of mucus, there appeared to be widespread tension emphysema with the left lung more affected than the right.

\section{Lobular Obstructive Emphysema : Aspiration of Vomitus}

Case 9. This boy was born at term, the birth weight being $7 \mathrm{lb} .4 \mathrm{oz}$. $(3,289 \mathrm{~g}$.). At the age of 11 weeks he developed an acute episode of vomiting. A few hours after the onset of this he was found in his cot collapsed and in obvious respiratory distress. He was admitted to hospital on May 9, 1956, exhibiting marked bronchospasm and with the chest fully inflated. He died shortly after admission.

Post-mortem examination (Dr. K. Rhaney) showed aspirated vomit throughout most of the bronchial tree, even to the smallest respiratory passages. Most of the lung tissue was acutely over-distended with air and the lungs did not collapse on opening the chest. Histological examination confirmed the emphysema of the lungs and showed no evidence of acute inflammation.

No doubt in this case the vomitus blocked the bronchi in such a way as to obstruct outflow of air but allowed some entry of air on inspiration.

\section{CYSTIC OBSTRUCTIVE EMPHYSEMA}

Cystic Obstructive Emphysema : Congenital or Acquired Cyst

Case 10. This boy was born six weeks prematurely on March 4, 1955, the birth weight being $3 \mathrm{lb} .12 \mathrm{oz}$. $(1,701$ g.). He fed poorly from birth but apart from that remained reasonably well for two weeks when he developed gastro-enteritis. This illness was complicated by pneumonia. Two weeks later he suffered a second attack of pneumonia from which he again made a satisfactory recovery.

At the age of $3 \frac{1}{2}$ months he had a third attack of pneumonia accompanied by a febrile convulsion, and was admitted to hospital. Radiological examination showed an air-containing cyst in the left lung in the posterior basal segment of the lower lobe (Fig. 12).

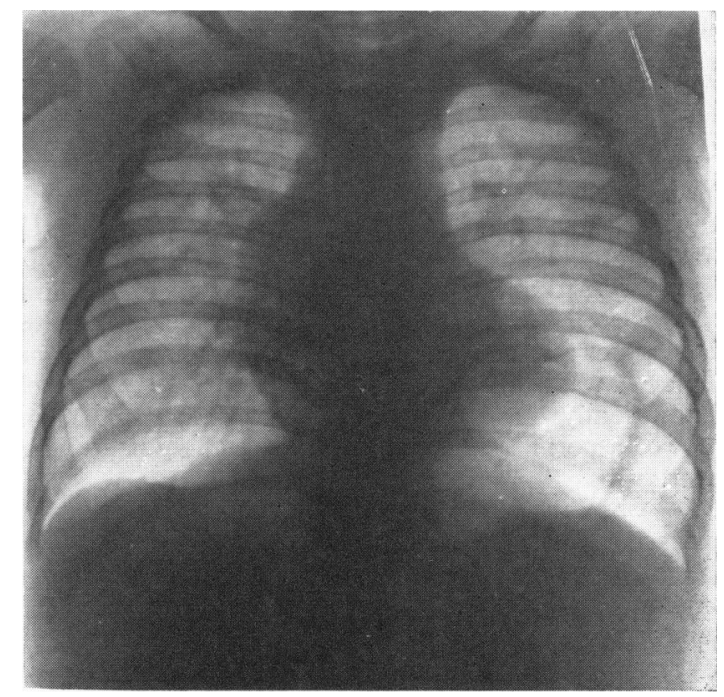

FIG. 12.-Case 10: Congenital or acquired cyst of the left lower lobe.

Clinically he was noted to be intermittently dyspnoeic, particularly on sitting up. The intercostal spaces were markedly indrawn and the costal margin receded on respiration. He had a persistent cough.

Intermittent respiratory embarrassment and indrawing of the chest persisted for four months. The cyst remained unaltered and it was decided to remove it. At operation on November 16, the lower lobe was found to be adherent to the paravertebral gutter and the diaphragm. The lung was freed and the lower lobe was found to contain a large cyst with collapse of surrounding lung tissue. The cyst was removed by resection (Mr. M. Fallon).

On recovery the remainder of the left lung expanded fully, and over a period of a fortnight all abnormal signs disappeared from the chest, except some costal indrawing.

On pathological examination (Dr. K. Rhaney) the cyst was found to be surrounded by lung tissue. A bronchus entered the cyst from the supero-medial aspect, running obliquely through the wall and entering the cavity under a crescentic valve-like fold in the external lining. Macroscopically there was no evidence of pneumonia. Histologically the cyst was found to be of bronchial origin, lined by pseudo-stratified ciliated columnar epithelium. 
Deep to the epithelium were bundles of smooth muscle fibres forming a complete layer round the cyst. A bar of cartilage was present in the cyst wall, the surrounding lung was collapsed, and in the hilar region there was some chronic inflammatory change.

It would be difficult to say whether this cyst was congenital and predisposed to recurrent pneumonia or whether it was the result of the attacks of pneumonia. The mechanism whereby it persisted seems clear, however, namely the valvular entry of the bronchus into its wall.

\section{Cystic Obstructive Emphysema : Post-pneumonic Pneumatocoeles}

Case 11. This girl was the third child of healthy parents. She was born at full term on May 7, 1953, her birth weight being $7 \mathrm{lb} .13 \mathrm{oz}$. $(3,544 \mathrm{~g}$.). On the second day she began to vomit and on the fourth day the vomitus was slightly bloodstained. An oesophageal hiatus hernia was suspected and on the sixth day a 'lipiodol' swallow was given. This showed that an oesophageal hiatus hernia was present, but during the examination some 'lipiodol' was aspirated into the lungs with the result that inadvertently bronchography was carried out. This showed a normal bronchial tree and the lung fields were clear.

She was nursed in the upright position and the vomiting lessened. She was discharged home on the 23rd day gaining weight satisfactorily. Eight days later she was readmitted to hospital. For the previous few days the vomiting had increased, she had become fevered and her respirations had become grunting and rapid. Physical examination showed a marked impairment of the percussion note over the upper half of the left lung, coarse crepitations over the posterior aspect of the left lung and some mediastinal displacement to the right. The white cell count was $27,000 /$ c.mm. Radiological examination of the chest showed consolidation of the left upper lobe with early formation of multiple bullae. She was given penicillin intramuscularly, 100,000 units four-hourly, and streptomycin, $0 \cdot 25 \mathrm{~g}$. six-hourly. After two days her condition remained unchanged and on this account she was given oxytetracycline, $50 \mathrm{mg}$. six-hourly by mouth, in place of the other antibıotics. From sputum obtained by a cough swab coagulase-positive staphylococci were cultured. The diagnosis made was that of staphylococcal pneumonia involving predominantly the upper lobe of the left lung.

Clinically she responded satisfactorily to oxytetracycline therapy but serial radiological examination showed the development of large emphysematous bullae within the substance of the left upper lobe. Over a period of several weeks these progressively increased in size showing evidence of positive pressure (Fig. 13). She developed an obvious asymmetry of the chest with ballooning of the upper part of the left thorax anteriorly, diminished respiratory movement on that side and costal margin recession. On percussion the area of deep cardiac dullness was absent. There was diminished air entry over the left lung.
This infant continued to be nursed in the upright position and despite the large bullae made very satisfactory progress with a normal weight gain and no cough. Her respiration rate varied between 30 and 70 per minute. The bullae persisted unchanged for a period of nine months. At the end of that time they began to diminish in size and disappeared entirely over a period of two months. She has remained well since, over a period of two years, and has had no further respiratory symptoms. The chest radiograph remains quite clear

\section{Combined Cystic Obstructive Emphysema and Lobar Obstructive Emphysema}

Case 12. A boy, the third child of healthy parents, weighed $6 \mathrm{lb} .1 \mathrm{oz}$. $(2,750 \mathrm{~g}$.) at birth. The birth had been normal at full term and there had been no post-natal asphyxia or respiratory difficulties.

When 7 weeks old he suffered from bronchitis and a week later was admitted to an infectious diseases hospital with gastro-enteritis. He was in hospital for three and a half weeks due to slow recovery and poor weight gain. During his stay in hospital his respiration rate rose suddenly one day to 80 per minute, remained at 50 to 80 per minute for two days and then returned to normal. This incident was accompanied by marked tachycardia.

On June 24, 1949, at the age of 6 months, he was admitted to hospital, having been vomiting for three days. He was noted to be only $50 \%$ of the expected weight but no respiratory signs were evident. During the first week his respiration rate was occasionally a little rapid. On the tenth day, following a feed, he developed marked tachypnoea accompanied by respiratory distress. At the same time there was an obvious fullness of the right side of the chest compared with the left; there was a hyperresonant percussion note over the right chest anteriorly and there was marked lower intercostal and costal margin recession, the thoracic cage staying fixed in the position of full inspiration. The breath sounds were diminished over the right lung and the apex beat was

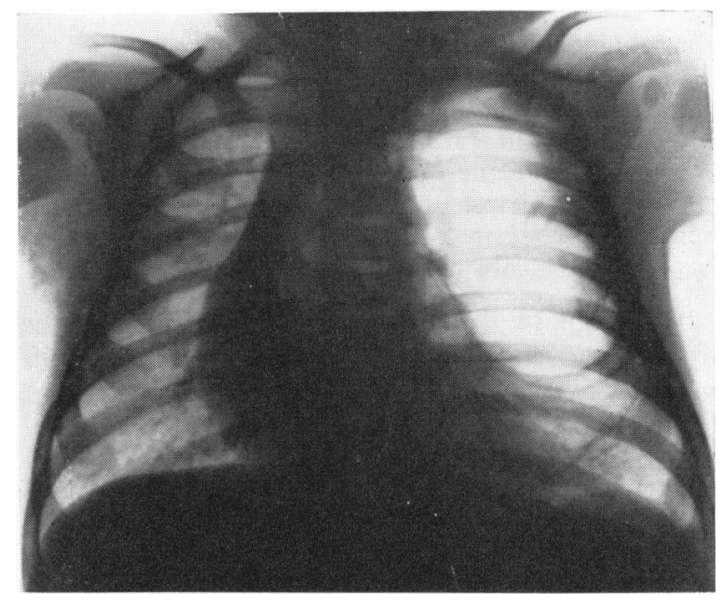

Fig. 13.-Case 11 : Fully developed post-pneumonic (staphylococcal) pneumatocoele. 
displaced to the left half an inch outside the midclavicular line. There was no cough or asthmatoid wheeze. The intradermal tuberculin test was negative up to a strength of $1 / 1,000$. Radiological examination confirmed the presence of obstructive emphysema of the right lung with abnormal radiotranslucency, herniation of the mediastinal pleura into the left hemithorax, cardiac displacement to the left, paradoxical movement of the mediastinum and diminished movement of the right diaphragm (Fig. 14). There appeared to be two air-

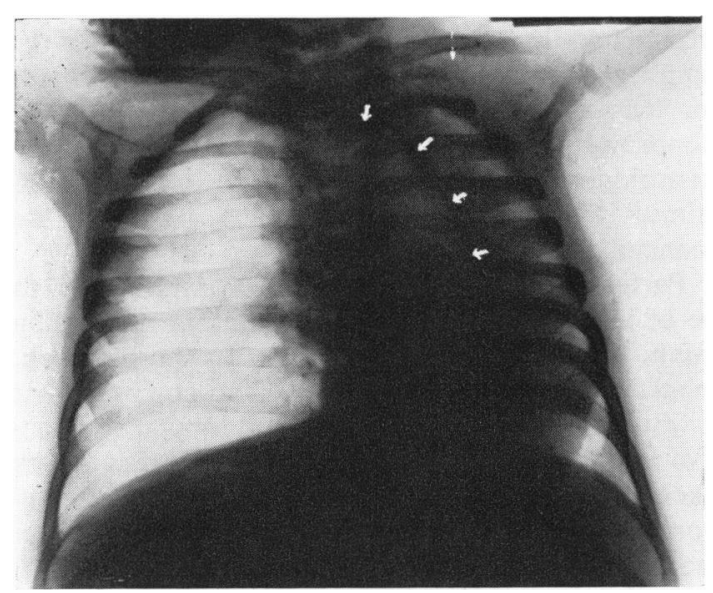

FIG. 14.-Case 12: Obstructive emphysema of the right lung with mediastinal pleural herniation.

containing cysts at the region of the right hilum. Thereafter tachypnoea (50 to 100 per minute) and respiratory embarrassment remained constant.

A few days later bronchoscopy was carried out (Mr. M. J. Gibson). The lumen of the right main bronchus was seen to be moon-shaped, apparently as a result of external pressure. Thereafter dyspnoea became progressively worse, the child's condition deteriorated and he died three weeks after admission to hospital.

At post-mortem examination (Dr. A. B. Goodall) the right lung was voluminous and occupied the whole of the right half of the thoracic cavity and part of the left half, its left anterior border being $2 \mathrm{~cm}$. to the left of the midline. The heart was displaced to the left, the right border of the heart being to the left of the midline. The left lung appeared small and compressed towards the posterior chest wall. The heart was normal and the ductus arteriosus closed. The innominate artery was very short and the right common carotid and subclavian arteries sprang almost directly from the aorta. The bronchial and intercostal arteries appeared to originate normally. A multilocular mucous cyst was present in the superior mediastinum on the left side behind the oesophagus and the trachea, the largest loculus of this cyst being about half an inch in diameter. On section the gross emphysema of the right lung was confirmed. There was no differentiation of the lung into lobes. At the hilar region within the lung substance there was a cyst $\frac{1}{2}$ in. in diameter and immediately above it a smaller cyst about half this size. These cysts were in close relationship to the right main bronchus, the bronchus passing across the posterior wall of the main cyst in its upper aspect. The cysts contained air. Inflation of the right main bronchus caused air bubbles to appear at a slit-like opening in the antero-lateral wall of the larger cyst. This opening was covered by a flap and the obliquity of the track into the cyst was such that the flap acted as a valve preventing egress of air from the cyst. The left lung was not normally lobulated and there was only a rudimentary interlobar fissure. Lymph nodes were not enlarged.

The spleen weighed $4 \mathrm{~g}$. and the liver $170 \mathrm{~g}$. The left kidney weighed $23 \mathrm{~g}$. and there was a normal left ureter. The right kidney and right ureter were absent. The right renal artery was also absent and there was no right ureteric orifice in the bladder. The pancreas appeared normal.

In this case there appeared to be a double valvular mechanism: an air-containing tension cyst at the hilum exerting pressure on the right main bronchus in such a way that valvular emphysema of the lung resulted.

\section{Discussion}

These cases are fairly gross examples of regional obstructive emphysema in infancy and illustrate a variety of pathological conditions in which this disorder may occur.

The classification suggested might be represented schematically in the following way:

REGIONAL OBSTRUCTIVE EMPHYSEMA

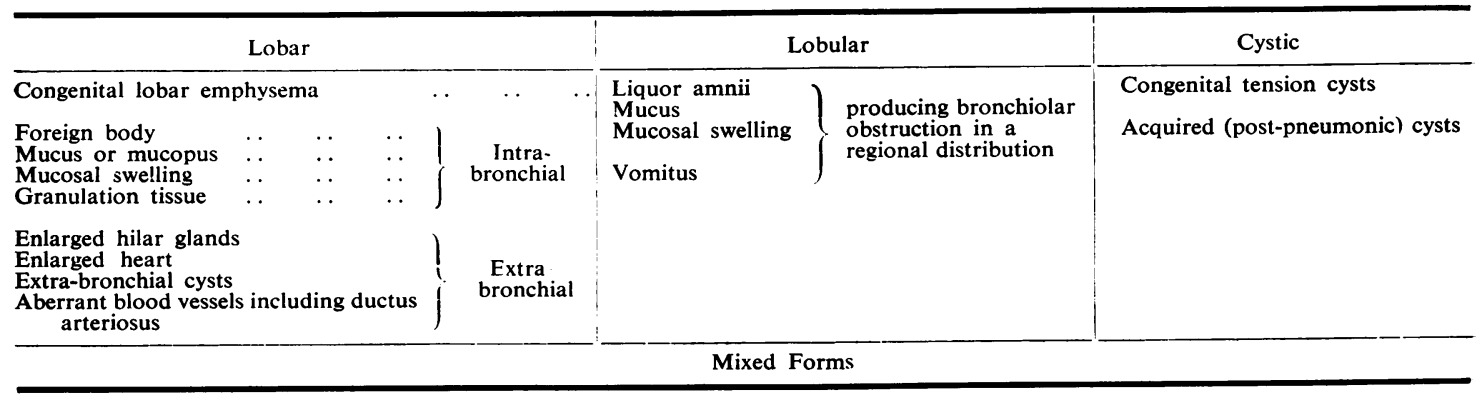


It is not suggested that every cause of regional obstructive emphysema is mentioned in the above classification but this classification could probably include within it all forms of regional obstructive emphysema whatever the cause.

\section{Diagnosis}

Clinically marked obstructive emphysema is likely to be associated with a bulging or 'blown' chest wall, with a resonant or hyper-resonant percussion note and with diminished air entry over the affected region. There may also be signs of mediastinal displacement and loss of cardiac dullness.

Radiologically obstructive emphysema is most likely to be confused with collapse associated with compensatory emphysema. In obstructive emphysema there is essentially evidence of positive pressure over-inflation of the affected part of the lung so that in grosser degrees there may be downward depression of the diaphragm, splaying of the ribs, anterior bowing of the sternum, mediastinal shift, mediastinal pleural herniation and paradoxical movement of the mediastinum on breathing. In Cases 2, 3 and 5 obstructive emphysema of one lobe was associated with secondary pressure collapse of the adjacent lobe. It is important that such a secondary collapse due to external pressure should be differentiated from primary collapse due to bronchial obstruction and accompanied possibly by some compensatory emphysema. As Robertson and James (1951) have pointed out, primary collapse, in contradistinction to secondary pressure collapse, is associated with elevation rather than depression of the diaphragm, and with a greater degree of radioopacity than a lobe subject to compression by an adjacent emphysematous lobe. The degree of emphysema in compensatory emphysema is at most only slight. Nor is compensatory emphysema ever associated with the ballooning and mediastinal herniation of obstructive emphysema. It seems to us that the radiological diagnosis of compensatory emphysema is made too often in infancy and childhood on the analogy of adult experience and without sufficient regard to the likelihood of obstructive emphysema at this age and to the factors which indicate its presence.

In its lesser degrees, obstructive emphysema may be difficult to demonstrate radiologically. The contrast between normal and emphysematous lung in regional obstructive emphysema does, however, make recognition easier then in generalized emphysema.

As obstructive emphysema is a functional condition dependent on active respiration, lungs affected by it may return to normal size after respiration ceases, and if the degree of over-inflation has been insufficient to exceed the elastic limit of the lung no abnormality may be revealed at necropsy (Carter, 1940).

\section{General Role of Obstructive Emphysema in Producing} Respiratory Symptoms in Infancy

Obstructive emphysema may be a much commoner factor in the genesis of respiratory embarrassment in infancy than is generally realized. Because of the difficulty of recognizing it in its minor degrees and at post-mortem examination, the significant role which it may play in respiratory disease in infancy may have been underestimated.

The extent of respiratory distress is no doubt proportional to the volume of lung affected by the emphysematous process and to the severity of the air tension within the lung. It may be, too, that abnormal pressure changes in the lung disturb the neurological control of respiration by virtue of stimuli transmitted via the vagus nerve in the manner of the Hering-Brauer reflex.

Particularly is obstructive emphysema likely to be of importance in the newborn infant and in the infant with respiratory infection. In the newborn, inhalation of mucus and liquor is so common that it would be surprising if widespread lobular obstructive emphysema did not frequently occur. It appears likely that spontaneous pneumothorax in the newborn infant, as seen in Case 6, results from such a mechanism (Silver, 1939). Davis and Stevens (1930) have demonstrated the presence of pneumothorax radiologically in almost $1 \%$ of 702 consecutive newborn babies and Solis-Cohen and Bruck (1934) in approximately $2 \%$ of 500 neonates. A much higher proportion must surely develop obstructive emphysema without going on to pneumothorax. Where spontaneous pneumothorax develops there are two possible routes by which the air can reach the pleural cavity from the pulmonary alveoli. It may rupture directly through the visceral pleura of the lung, or it may travel indirectly, rupturing first into the interstitial tissue (Howie and Weed, 1957), tracking thence to the root of the lung (Macklin, 1939) and rupturing through the mediastinal pleura which yields more easily than does the visceral pleura (Hamman, 1939). The latter appears to be the common route. In infection, too, conditions predisposing to obstructive emphysema are likely to be present. Inflammatory swelling and secretions are likely to block the interalveolar pores and the smaller bronchi and bronchioles are likely to be partially obstructed by swelling of their lining mucosa and by inflammatory exudate. From the clinical point of view the over-inflation and bulging of the thoracic cage and the hyper-resonant percussion note seen frequently in respiratory infection in infants are in keeping with the view that in such cases obstructive emphysema is a common and significant cause of disordered respiratory function. 
Acute bronchiolitis, in particular, creates conditions favourable for the occurrence of generalized obstructive emphysema (Nelson and Smith, 1945).

In the adult, in contradistinction, obstructive emphysema appears to be an uncommon occurrence, although it has been reported to have resulted from mucous plugs and from carcinoma obstructing the main bronchi (Maxwell, 1940). The infant seems to be much more prone to it and the narrow lumen of the bronchi and greater elasticity of the bronchial walls may be relevant factors. During the period of infancy, too, congenital anomalies of the bronchi are likely to reveal themselves. The possibility of their occurrence should be borne in mind in any infant who exhibits episodic tachypnoea, intercostal indrawing or respiratory embarrassment.

\section{Lobar Obstructive Emphysema}

Although in some cases of lobar emphysema, as for example with a foreign body, the check-valve mechanism described by Jackson is clearly evident this is not always so.

In congenital lobar emphysema no obvious valvular mechanism may be evident (White-Jones and Temple, 1954; Holzell, Bennett and Vaughan, 1956). In other cases deficiency of the cartilaginous rings of the bronchial wall similar to that seen in Case 1 has been described (Overstreet, 1939; Fischer, Tropea and Bailey, 1943; Shaw, 1952) and it is postulated that in these undue flaccidity of the bronchial wall allows it to collapse or become distorted during expiration in such a way as to cause obstruction to the egress of air. An association between mucous membrane folds and deficient bronchial cartilage has been reported (Fischer et al., 1952) and mucosal folds alone have been held responsible for obstructive emphysema (Robertson and James, 1951). External pressure on a bronchus by an aberrant artery and by a patent ductus arteriosus has been reported (Robertson and James, 1951; Fischer et al., 1952). Clark, Nairn and Gowar (1956) have brought forward evidence that congenital bronchostenosis may occur and act as a check valve. The bronchial obstruction which in Case 11 resulted from external pressure by a congenital cyst which was itself subject to obstructive emphysema appears to be a unique mechanism.

In congenital lobar emphysema symptoms of dyspnoea, tachypnoea and cyanosis may be present from birth. Frequently they develop or recur within a few days or weeks of birth. Distress may be acute and early recognition may be life saving.

Barsby (1941) reported three cases of obstructive emphysema resulting from pressure by tuberculous hilar glands, and at that time could find only 16 other reports of this condition in the literature. The majority of cases occurred during the first year of life.

Whether or not operative treatment is employed in lobar obstructive emphysema must depend on the cause and nature of the valvular mechanism and the severity of the emphysema. In Case 1, not operated on, congenital lobar emphysema proved rapidly fatal. In Case 2 operative treatment was successful. In Case 3 spontaneous recovery seems likely. Several reports on the value of resection of affected lobes in congenital lobar emphysema have appeared (Leahy and Butsch, 1949; Robertson and James, 1951; Williams, 1952; Fischer et al., 1952; Holzel et al., 1956). Sloan (1953), however, reported four patients who had residual symptoms after lobectomy. Two of these showed radiological evidence of the further development of emphysema in other parts of the lung following operation. Cases of congenital lobar emphysema can recover spontaneously (Caffey, 1953; Holzel et al., 1956). Holzel et al. have described a child with congenital lobar emphysema showing symptoms from birth and not operated on who gradually improved as he grew older. At 5 years of age he was in good health and radiologically there was minimal evidence of emphysema. Thus, although resection of the affected lobe in congenital lobar emphysema may be a life-saving measure and is probably indicated in severe cases, it does not follow that operation should be invariably undertaken. The extent and frequency of respiratory embarrassment will probably be the determining factors. Deflation of the lung can be carried out as an immediately pre-operative measure by the introduction of a needle through the chest wall, and in acute respiratory embarrassment this may be a necessary emergency procedure. Although we used this procedure successfully in Case 2 it has not always been found to be of benefit.

In the acquired forms of lobar obstructive emphysema except for that produced by foreign bodies there is probably less scope for operative treatment. A foreign body in a bronchus which cannot be dislodged by coughing or posturing is a clear indication for bronchoscopic removal. Tuberculous granulation tissue which can be clearly visualized on bronchoscopy and which is causing respiratory distress in virtue of obstructive emphysema should probably be removed (Graham and Hutchison, 1947). Thoracotomy for the removal of tuberculous hilar glands exerting pressure on bronchi and causing severe obstructive emphysema may be an operative emergency (Macpherson, 1957). Where, as in Case 6, mucopus and swelling of the mucous membrane are responsible for obstruction, appropriate antibiotic therapy will be the most important factor in treatment. 


\section{Lobular Obstructive Emphysema}

Considering the various types of emphysema quantitatively, lobular emphysema is almost certainly the most commonly occurring for the reason that aspiration into the lungs in the newborn and infection and bronchospasm in infancy are so common. When severe and massive the clinical features of pulmonary over-inflation resulting from lobular emphysema may be obvious enough. In its lesser forms, however, lobular obstructive emphysema may be less evident clinically. Yet arguing by analogy from the severe generalized lobular obstructive emphysema seen in acute bronchiolitis of infancy and from the severe regional lobular obstructive emphysema present in cases such as 7 and 9 reported here, it is likely that any obstruction of smaller bronchi or bronchioles in infancy by aspirated fluid, secretions, or swelling of the lining mucosa may result in lobular emphysema of varying degree. The development of obstructive emphysema in any lobule is likely to interfere to a greater or lesser extent with its functional capacity.

\section{Cystic Obstructive Emphysema}

Conway (1951) has described varieties of lung cyst and discussed the aetiology in the light of the cases which he reported and the literature on the subject. $\mathrm{He}$ accepts that congenital cysts can undoubtedly occur but concludes that the majority of lung cysts seen after the first few days of life are acquired. Clark et al. (1956) have recently described the successful removal of a lung containing multiple congenital cysts. Successful removal of congenital lung cysts has also been described by Fischer $e t$ al. and by Conway. There appears little doubt that the congenital cysts in Case 12 were primarily responsible for the fatal lobar emphysema from which the patient suffered, and an attempt at operative removal should have been made.

Post-pneumonic pneumatocoeles, which constitute the main differential diagnosis from congenital cysts usually have a good prognosis and tend to disappear spontaneously. Where, as is so frequently the case, congenital cysts or acquired post-pneumonic cysts are subject to tension emphysema the valvular mechanism seems usually to be that which occurred in Case 10. The tangential opening of the supplying bronchus into the cyst results in a slit-like or flap-like mouth which acts as a valve and whose valvular nature tends to be preserved by the pressure in the cyst (Fig. 1). There is thus a vicious cycle of events maintaining the obstructive emphysema within the cyst. Some factor suddenly breaking this cycle may be responsible for the sudden disappearance of, for example, a post-pneumonic pneumatocoele. Clark et al. (1956) have suggested that multiple congenital cysts of the lung can result from the presence of both bronchiolectasis and a check-valve obstruction of the bronchus. In the presence of the increased tension within the lung resulting from the check valve the bronchiolectatic areas distal to the valve expand to form cysts.

\section{Mixed Forms of Regional Obstructive Emphysema}

Although it is convenient and possibly useful to classify obstructive emphysema into three main types these may not occur in a 'pure' form. Case 12 illustrates a 'mixed' form in which cystic and lobar emphysema were combined. The assertion of Clark et al. (1956) that areas of congenital bronchiolectasis may become cystic in the presence of a check-valve obstruction of a main bronchus causing lobar emphysema suggests another mixed form.

\section{Summary}

It is suggested that regional obstructive emphysema can be classified as lobar, lobular or cystic with subsidiary groupings dependent on pathogenesis.

A series of cases in infants is reported illustrating varieties of regional obstructive emphysema. These are classified according to the scheme outlined above.

Diagnosis is discussed and the important role of obstructive emphysema in the production of respiratory symptoms in infancy is emphasized.

The mechanism of production of the various forms of regional obstructive emphysema and their treatment is considered.

\section{REFERENCES}

Barsby, B. (1941). Lancet, 1, 627.

Caffey, J. (1953). Pediatrics, 11, 48

Carter, R. A. (1940). Radiology, 35, 391

Clark, N. S., Nairn, R. C. and Gowar, F. J. S. (1956). Arch Dis. Childh., 31, 358

Conway, D. J. (1951). Ibid., 26, 504.

Davis, C. H. and Stevens, G. W. (1930). Amer. J. Obstet. Gynec., $20,73$.

Fischer, C. C., Tropea, F. and Bailey, C. P. (1943). J. Pediat, $23,219$.

Graham, S. G. and Hutchison, J. H. (1947). Arch. Dis. Childh., 22, 162.

Hamman, L. (1939). Ann. intern. Med., 13, 923.

Heinbecker, P. (1927). J. clin. Invest., 4, 459.

Holzel, A., Bennett, E. and Vaughan, B. F. (1956). Arch. Dis. Childh. 31, 216.

Howie, V. M. and Weed, A. S. (1957). J. Pediat., 50, 6.

Jackson, C. (1930). J. Amer. med. Ass., 95, 639.

Leahy, L. J. and Butsch, W. L. (1949). Arch. Surg. (Chicago), 59, 466.

Macklin, C. C. (1939). Arch. intern. Med., 64, 913.

Macpherson, A. M. C. (1957). In Symposium of Tuberculosis, ed. F. R. G. Heaf. London.

Maxwell, J. (1940). Brit. med. J., 1, 520

Nelson, W. E. and Smith, L. W. (1945). J. Pediat., 26, 36.

Overstreet, R. M. (1939). Amer. J. Dis. Child., 57, 861.

Reinberg, S. A. (1925). Brit. J. Radiol., (B.I.R. Sect.), 30.451.

Robertson, R and James, E. S. (1951). Pediatrics, 8, 795.

Shaw, R. R. (1952). Ibid., 9, 220.

Silver, H. B. (1939). Amer. J. Dis. Child.. 57, 907.

Sloan, H. (1953). J. thorac. Surg., 26, 1.

Solis-Cohen, L. and Bruck, S. (1934). Radiology, 23, 173

Van Allen, C. M. and Soo, Y. C. (1933). J. clin. Invest., 12, 171

White-Jones, R. H. and Temple, L. J. (1954). Arch. Dis. Childh., 29, 248 .

Williams, M. H. (1952). J. thorac. Surg., 24, 522. 